\title{
A cien años del inicio de la Gran Guerra: brote de triquinosis en marinos del Dresden en Chile. Crónica de una infección anunciada
}

\author{
Italo Fernández y Alejandro Mihovilovich
}

Laboratorio de Parasitología. Departamento de Microbiología Facultad de Ciencias Biológicas,

Universidad de Concepción, Concepción (IF)

Biblioteca Municipal de Concepción "José Toribio Medina"

Declaración de conflictos de interés: No existen conflictos de interés.

Fuente de financiamiento Facultad de Ciencias Biológicas. Universidad de Concepción. Recibido: 27 de diciembre de 2016

Correspondencia a: Italo Fernández F. itfernan@udec.cl

\section{Introducción}

$\mathrm{E}$ n 2014 se cumplieron cien años del inicio de la Primera Guerra Mundial. Si bien, el escenario principal de las operaciones bélicas se ubicó a miles de kilómetros de distancia, hubo variados acontecimientos que vincularon a Chile con esta conflagración mundial. La bibliografía respectiva recoge contribuciones principalmente relacionadas con aspectos de carácter militar por lo que, a propósito de esta conmemoración, hemos creído conveniente recordar la ocurrencia de un brote de triquinosis en marinos pertenecientes a la tripulación del SMS Dresden, buque alemán de activa participación en el conflicto, y que se encontraban recluidos en la isla Quiriquina, región del Bío Bío, Chile. El objetivo de este trabajo es refrescar un hecho relativamente desconocido dentro del contexto bélico y, por otra parte, revisar los aspectos epidemiológicos que determinaron su ocurrencia y que, incluso hasta hoy, persisten en la zona.

\section{Contexto histórico}

El 28 de julio de 1914 se inició la Primera Guerra Mundial entre las potencias centrales (Imperio alemán, Imperio otomano, Bulgaria y austro-húngaro) y la triple entente (Reino Unido, Francia, Imperio ruso, Italia y, posteriormente, Japón y Estados Unidos). Las hostilidades ocurrieron principalmente en Europa y en ambientes terrestres. En el mar, la guerra se caracterizó por el intento de bloqueo marítimo por parte de ambos bandos, representados principalmente por la Armada Imperial Alemana y la Marina Real Británica ${ }^{1}$.

\section{El Dresden y su periplo durante la guerra}

El S.M.S. Dresden (Figura 1), era un crucero ligero alemán que fue botado al mar en 1906. Dentro de sus características principales constaba de tres chimeneas, 118 metros de eslora y turbinas Parsons que le otorgaban la capacidad de alcanzar los 28 nudos de velocidad. La tripulación la componían 371 marinos al mando del Capitán de Navío Fritz Lüdecke (Figura 2) 2 . Al inicio de la guerra, el barco se encontraba en el mar de China y se dirigió hacia Rapa Nui con el objetivo de reunirse con el resto de la Flota alemana, liderada por el Almirante Maximilian von Spee. Desde ahí zarparon rumbo al Cabo de Hornos con el fin de interceptar y romper rutas de aprovisionamiento entre América y Europa. Participó en la Batalla de Coronel, el 1 de noviembre de 1914, donde la flota alemana resultó victoriosa. Sin embargo, en la batalla de las islas Malvinas, ocurrida el 8 de diciembre del mismo año, la flota alemana fue hundida casi en su 


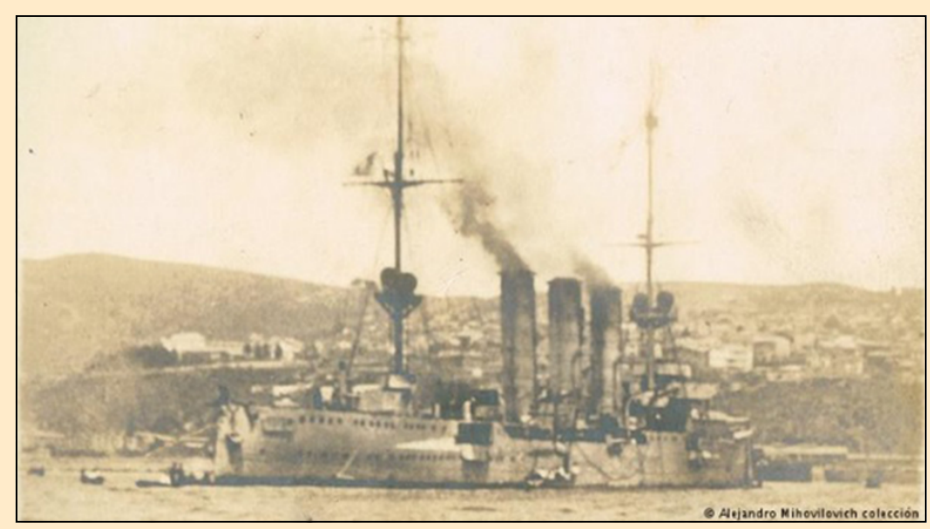

Figura 1. Crucero S.M.S. Dresden (Fotografía colección personal Profesor Alejandro Mihovilovich G).

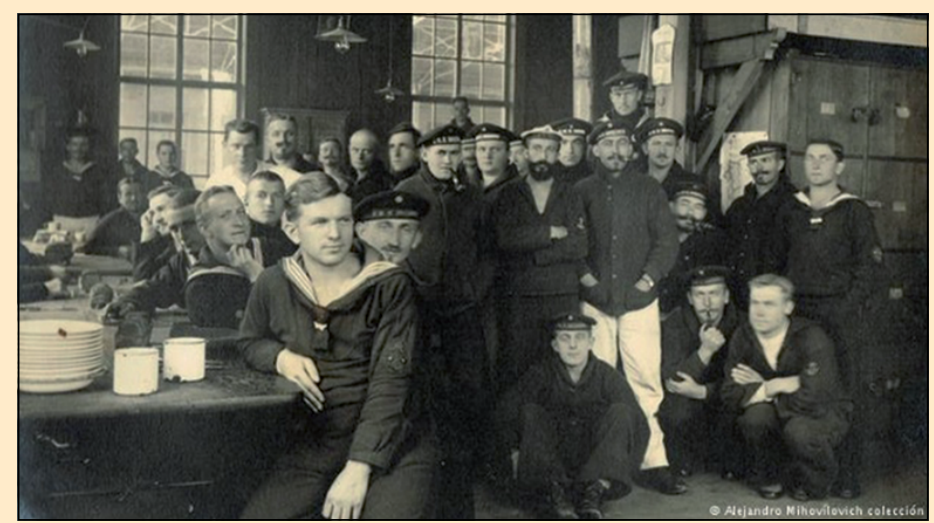

Figura 3. Marineros del Dresden en Isla Quiriquina (1915-1918). (Fotografía colección personal Profesor Alejandro Mihovilovich G.)

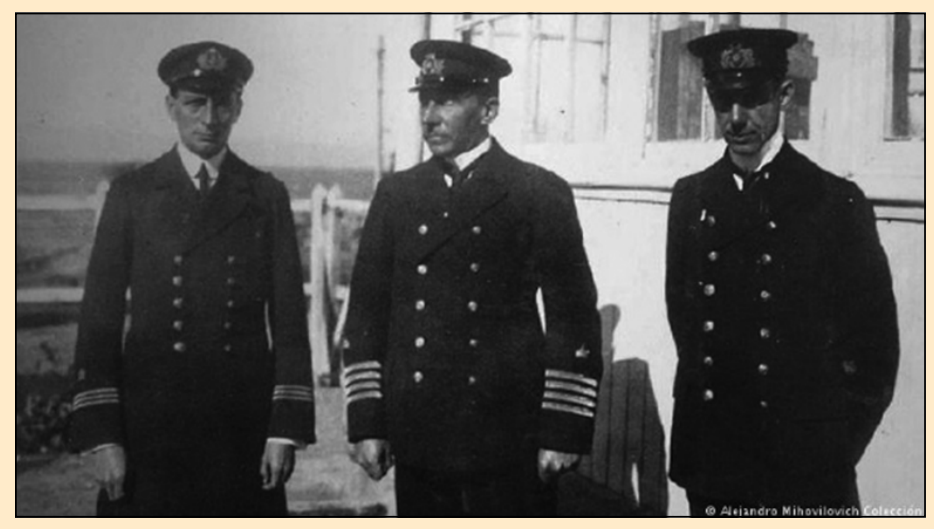

Figura 2. Capitán Fritz Lüdecke (al centro) acompañado del jefe del Apostadero Naval de Talcahuano (izquierda) y del Segundo Comandante del Dresden Wilhelm Canaris (Fotografía colección personal Profesor Alejandro Mihovilovich G.)

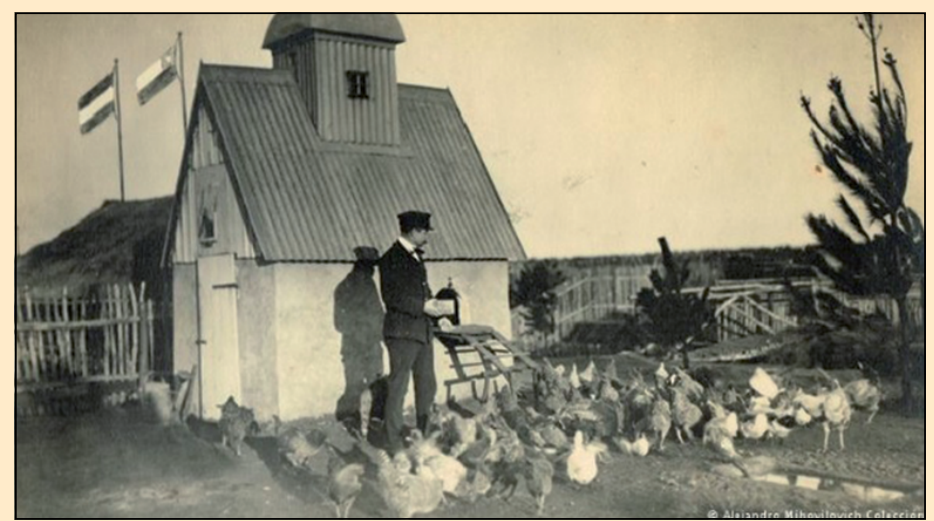

Figura 4. Granja de animales construida por marineros del Dresden en Isla Quiriquina (1915-1918). (Fotografía colección personal Profesor Alejandro Mihovilovich G.) totalidad por la armada inglesa. El Dresden fue el único navío sobreviviente debido a la mayor velocidad que le otorgaban sus turbinas, logrando escapar ${ }^{3}$. Con el fin de evitar su captura, por sus escasas reservas de víveres y carbón, además de la necesidad de reparar daños, el buque se escondió hábilmente en los canales de la Patagonia chilena. En estos parajes la tripulación pudo reparar las averías y surtirse de recursos alimenticios merced a la pesca, caza, fuentes de agua natural y la ayuda de colonos alemanes residentes en Chile ${ }^{4}$. A fines de febrero de 1915 zarpó con el objetivo de encontrarse con un barco alemán que lo abasteciera de víveres y combustible en alta mar y emprender la ruta de los veleros hacia Oceanía. Sin embargo, hubo necesariamente de navegar hacia el Archipiélago de Juan Fernández para reabastecerse de carbón. Fondeó en la bahía de Cumberland de la Isla Más a Tierra, el día 9 de marzo de 1915, permaneciendo ahí durante cinco días. En la mañana del 14 de marzo fue atacado sorpresivamente por los buques ingleses Kent,
Glasgow y Orama, que lo habían estado persiguiendo desde los mares australes. Incapaz de hacer frente a la agresión, producto de la escasez de carbón, el capitán Lüdecke decidió hundir el barco, mientras ordenó a la tripulación desembarcar en la isla. Ocho marinos alemanes perecieron en el combate. Los quince heridos más graves fueron trasladados a Valparaíso en el Orama, mientras que el resto de la tripulación fue embarcada el 25 de marzo a bordo de la Esmeralda y el Zenteno, navíos de la Armada chilena, y transportados hacia la isla Quiriquina (Talcahuano, centro-sur de Chile) ${ }^{5}$.

\section{Reclusión en isla Quiriquina y presentación del brote}

En la isla Quiriquina $\left(36^{\circ} 37^{\prime} 33^{\prime \prime} \mathrm{S} 73^{\circ} 03^{\prime} 39^{\prime \prime} \mathrm{O}\right)$, ubicada a 11 kilómetros del puerto de Talcahuano y a 23 kilómetros de la ciudad de Concepción, los marinos 
alemanes quedaron recluidos forzosamente por el gobierno de Chile debido al deseo de éste de conservar la neutralidad del país durante el conflicto bélico (Figura 3). La mayoría de ellos ocupó el oficio que tenía antes de entrar a la marina. De esta forma, realizaron distintas funciones: construyeron casas, fabricaron artesanías de madera y hierro, formaron una banda musical, imprimieron un periódico, incluso remodelaron una planta de agua potable. La alimentación de la tripulación fue provista a partir de sus propios cultivos, un invernadero (construido por ellos mismos) y la producción de animales (Figura 4).

Los primeros casos del brote de triquinosis comenzaron a ser atendidos a finales de septiembre de 1915 y la fuente de infección fue por consumo de carne de cerdo. No se tiene claridad de cuántos fueron los marinos afectados ni existen registros de los síntomas que desarrollaron, aunque se indica que sesenta presentaron sintomatología sugerente de triquinosis que obligó a su internación en el Sanatorio Alemán de Concepción, en donde hubo que lamentar un caso fatal ${ }^{6}$, que resultó ser un suboficial de la tripulación fallecido el 1 de octubre ${ }^{7}$.

\section{Discusión}

En Chile y, particularmente la región del Bío Bío, la triquinosis humana tiene carácter endémico debido a que desde el siglo XIX se han registrado epidemias y casos aislados $^{8}$. De hecho, particularmente en la zona de Concepción era tan frecuente la ocurrencia de brotes epidémicos de triquinosis que el parasitismo era conocido como "fiebre penquista" aludiendo al gentilicio de la población que habitaba esta región ${ }^{9}$. Cabe notar que el descubrimiento y descripción de casos humanos de triquinosis ocasionados por el consumo de carne de cerdo, sólo había sido comunicado por Zenker en $1860^{10}$. Además, la transmisión de Trichinella spiralis habitualmente ha ocurrido en el contexto del ciclo doméstico o zoonótico del parásito, donde participan principalmente el ser humano, cerdos y roedores, estos últimos considerados su reservorio natural ${ }^{11}$. La crianza casera del cerdo era una actividad que se practicaba frecuentemente dentro del área urbana de Concepción en esos días. Así, el cerdo se criaba y "engordaba" hasta su posterior faenamiento, usualmente en meses de otoño e invierno, preservando sus carnes mediante el ahumado o secado al medio ambiente y fabricando subproductos como las longanizas, embutidos que pudieron servir como sustento alimenticio y/o fuente de ingresos, mediante su comercialización ${ }^{12}$.

En la zona de Concepción, las condiciones de salubridad a fines del siglo XIX y principios del siglo XX podrían haber favorecido la ocurrencia de brotes epidémicos de triquinosis. Existe constancia de numerosas deficiencias en la disposición de desechos, basuras y aguas servidas así como la presencia de numerosos roedores, todo esto agravado por la ausencia de red de agua potable. Además, numerosas crianzas de cerdos artesanales ("chancherías") funcionaban en malas condiciones higiénicas y de manera clandestina dentro del radio urbano de la zona ${ }^{13}$, contraviniendo reglamentos municipales. En este sentido, desde 1901 la ciudad contaba con el "Desinfectorio Público", entidad que dependía de la Municipalidad y en donde se efectuaban revisiones de las carnes para determinar si podían ser destinadas a la venta pública, al igual que el Matadero Municipal. Por lo tanto, la presencia de numerosas chancherías que comercializaban la carne en forma clandestina y que funcionaban sin control sanitario constituyeron el ambiente propicio para que el ciclo de $T$. spiralis persistiera en el tiempo.

Respecto del brote, se informó de sesenta afectados, dentro de los cuales diez cursaron con sintomatología intensa. En 1915, fruto de los numerosos casos analizados desde los tiempos de la "fiebre penquista", se tenía certeza del cuadro clínico de este parasitismo por lo que su diagnóstico no fue especialmente dificultoso. El tratamiento, salvo una excepción, fue favorable. La confirmación diagnóstica de triquinosis fue efectuada en base a la demostración de las larvas en el cadáver del único fallecido ${ }^{6}$.

Las graves consecuencias de este brote motivaron al capitán Lüdecke a enviar una nota, apoyada por el Cónsul del entonces Imperio germánico, a las autoridades municipales de la época, fechada el 18 de octubre de 1915, con el fin de iniciar una investigación. Como consta en las actas de la Municipalidad de Concepción ${ }^{7}$, el alcalde respondió a la misma señalando que la causa de la infección fue la venta de embutidos por parte de carniceros alemanes de Concepción y que habían sido preparados a partir de carne de cerdos de "mala procedencia" debido a que no habían sido faenados en el Matadero Municipal de la ciudad. El diagnóstico confirmatorio de triquinosis lo efectuó el médico veterinario municipal en cecinas que los marinos alemanes aún mantenían en su poder. La indagatoria, además, permitió concluir que estos carniceros suministraban semanalmente carne y embutidos, y que los iban a dejar directamente a la isla. Paradojalmente en 1915, la alcaldía había tomado una serie de medidas para controlar el faenamiento y venta clandestina en diversas "chancherías" que funcionaban en la ciudad, debido a constantes denuncias estampadas por la ciudadanía que daban cuenta de las malas condiciones de salubridad y nulo control en que se ejercían dichas actividades. La inspección de estos lugares, lideradas por el médico veterinario municipal, constató el faenamiento de estos animales en malas condiciones de salubridad y, tras un posterior análisis en el Matadero Municipal, muchos estaban infectados con triquinosis. Producto de esto y a instancias de la alcaldía, en los primeros días de agosto 
de 1915 la justicia procesó a las personas involucradas y aplicó multas, incluso decretando la clausura de estos lugares. Curiosamente, las multas y castigos fueron publicitados con el fin de que la ciudadanía tomase las precauciones correspondientes. Lamentablemente, uno de los carniceros multados apareció como responsable del suministro de los embutidos a los marineros del Dresden, dos meses después.

La revisión de estos antecedentes nos permite concluir que en la infección triquinósica de estos marineros se confabularon una serie de hechos que favorecieron su ocurrencia. Por un lado, las deficientes condiciones higiénicas de la ciudad de Concepción, que la convertían en un lugar propicio para generar el ciclo zoonótico del parasitismo y la presencia de "chancherías", muchas de las cuales faenaban en forma clandestina. Por otra parte, la presencia de comerciantes inescrupulosos que, aun teniendo prohibición de operar, realizaban faenamiento y venta clandestina de carne o cecinas no inspeccionados. Por último, el habitual consumo de carne de cerdo por parte de los tripulantes del Dresden, del cual existe constancia en su periplo por las costas de Chile, probablemente cruda o insuficientemente cocida. El hilo conductor que hilvanó estas situaciones fue el régimen de reclusión de los marinos que, si bien fue forzoso, se hizo parcial en la práctica pues podían salir al continente con el compromiso de regresar. De esta forma, miembros de la tripulación tomaron contacto con la colonia alemana residente, hecho que permitió vincularlos con los carniceros que vendían estos animales. A su vez, el desconocimiento de las sanciones aplicadas a estos carniceros, probablemente por la barrera del idioma, terminaron por condenarlos a sufrir la infección añadiendo un tinte dramático adicional a su forzosa estadía en la región.

Tal alarma pública causó la continua ocurrencia de grandes brotes epidémicos de triquinosis, incluyendo el de los marineros del Dresden, que dicha situación pudo haber influido en la conducta de los habitantes de la zona que, a través de los años, adquirieron un conocimiento empírico que obligaba a cocer rigurosamente la carne de cerdo. De hecho, en la actualidad, a pesar de no existir obligatoriedad de inspección sanitaria a aquellos cerdos faenados en forma domiciliaria, la población rural comúnmente extrae trozos de carne del animal y los lleva a inspeccionar a consultas médico-veterinarias privadas. Esta situación, además, del mejoramiento de las condiciones de salubridad de la ciudad, estimuladas por la acción educadora de la Sociedad Médica de Concepción, la existencia del Desinfectorio Público y la regularización de la técnica de triquinoscopia como método de diagnóstico oficial en mataderos (a partir de 1947) contribuyeron a disminuir paulatinamente la ocurrencia de brotes epidémicos en la zona. Así, ya entre 1935 y 1937 la triquinosis porcina diagnosticada en el Matadero Municipal de Concepción alcanzó rangos de frecuencia que oscilaron entre 0,28 a $0,3 \%{ }^{6}$. Posteriormente, se encontró $7,8 \%$ de Rattus norvegicus infectados con $T$. spiralis que habían sido capturados en cuatro sectores de la ciudad de Concepción ${ }^{14}$. Más recientemente, se informó de 2,3\% de seroprevalencia de triquinosis humana ${ }^{15}$ y se demostró una frecuencia de $2 \%$ de triquinosis en personas fallecidas y autopsiadas por diferentes causas en la Provincia de Concepción ${ }^{16}$. Por último, en el período 2009 a 2013 la presentación de triquinosis humana en la región alcanzó una tasa de incidencia de 0,05 por cada 100.000 habitantes ${ }^{17}$. Si bien, esta tendencia al descenso se ha mantenido a través de los años, en la actualidad todavía persisten algunos factores epidemiológicos que han favorecido la ocurrencia de brotes epidémicos y casos aislados de triquinosis humana en la región. Las principales causas siguen siendo la ingestión de carne o subproductos de cerdo no inspeccionados y a su venta clandestina a la población ${ }^{18,19}$ debido a que la crianza artesanal del cerdo persiste como costumbre muy arraigada en la población rural de la región.

Nuestro propósito fue refrescar la ocurrencia de un brote epidémico de triquinosis de especial connotación histórica, dada la conmemoración de los cien años del inicio de la Primera Guerra Mundial. La revisión de los antecedentes disponibles da cuenta de la persistencia de algunos factores epidemiológicos que favorecen la presentación de este parasitismo en la zona.

\section{Epílogo}

En la Isla Quiriquina, los marinos permanecieron recluidos hasta el final de la guerra. Muy pocos escaparon, entre los que se cuenta el teniente de navío Wilhelm Canaris, de posterior figuración como jefe de la Abwehr en el período nazi, durante la Segunda Guerra Mundial (Figura 2). Alrededor de 60 marinos se quedaron a vivir para siempre en Chile y se insertaron en la comunidad alemana residente, algunos de los cuales, posteriormente, se destacaron por su aporte a la comunidad.

\section{Resumen}

A propósito de la conmemoración de los cien años del inicio de la Primera Guerra Mundial, se revisa la ocurrencia de un brote epidémico de triquinosis en tripulantes del SMS Dresden, buque alemán que participó en esa conflagración mundial. Este suceso, acaecido en septiembre de 1915, ocurrió mientras los marinos se encontraban forzosamente recluidos en la Isla Quiriquina, Región del Bío Bío, Chile. La causa del brote fue el consumo de embutidos fabricados a partir de carne de cerdo infectada con el parásito Trichinella spiralis e involucró a 60 mari- 
neros, de los cuales hubo un caso fatal. La revisión de los aspectos epidemiológicos que favorecían la presentación de triquinosis humana en la época permite concluir que, la crianza porcina en malas condiciones higiénicas, la venta clandestina de carnes y subproductos sin inspección sanitaria y la barrera idiomática fueron determinantes en la ocurrencia del brote epidémico.

\section{Referencias bibliográficas}

1.- Balaresque J. Operaciones Navales en el Cono Sur de América durante la Primera Guerra Mundial. Trabajo de Investigación IX Jornada de Historia Militar "El Ejército de Chile en el contexto de la Primera Guerra Mundial" 2014. http://www.acanav.mil.cl/agn/documentos/trabajos_ investigacion/2014/Trabajo_de_investigacion_03.pdf (accedido el 2 de septiembre de 2016).

2.- Lazcano D. SMS Dresden. Historia en imágenes del crucero alemán. RIL Editores, $1^{\text {era }}$ edición, Santiago de Chile 2010.

3.- Bravo G. La Primera Guerra Mundial en la Costa de Chile. Altazor, Viña del Mar 2005.

4.- Parker M T. Tras la estela del Dresden. Ediciones Tusitala, Santiago de Chile 1987.

5.- Johnson C. El tesoro del Dresden. Rev Marina Chile 1999; $116(6): 1-8$

6.- Wilhelm O, Ruiz del Río A. Contribución al estudio de la triquinosis en Chile (Primera Nota preliminar). Bol. Soc. Biol. Concepción (Chile) 1938; 12: 35-8.

7.- Municipalidad de Concepción. Nota de excesos al Sr. Cónsul del Imperio Germánico ante el deceso de un Suboficial por consumo de carne con triquinosis en el buque Dresden. Acta Municipalidad de Concepción 1915-1916.

8.- Alcaíno H. Antecedentes sobre triquinosis en Chile.
Monografía Med Vet 1984; 3: 29-42.

9.- Wilhelm O. Historia de la medicina penquista. An Esc Med (Concepción) 1962; 4: 103-29.

10.- Neghme A. Zenker y el descubrimiento de la triquinosis. Bol Chil Parasitol 1960; 15 (4): 84-5.

11.- Atías A. Parasitología Médica. Publicaciones Técnicas Mediterráneo, Santiago de Chile 1998.

12.- Acha P, Szyfres B. Zoonosis y enfermedades transmisibles comunes al hombre y a los animales. Volumen III. Organización Panamericana de la Salud Estados Unidos 2003.

13.- León M. Estudios sobre la "Capital del sur": ciudad y sociedad en Concepción 1835-1930. Ediciones del Archivo Histórico de Concepción, Concepción 2015.

14.- Ruíz del Río A. Contribución al estudio de las enfermedades parasitarias humanas transmitidas por las ratas en Concepción. Bol. Soc. Biol. Concepción (Chile) 1939; 13: 47-82.

15.- Contreras M C, Schenone H, Sandoval L, García M. Epidemiología de la triquinosis en Chile. Estudio de prevalencia mediante reacciones inmunodiagnósticas. Bol Chil Parasitol 1994; 49: 73-4.

16.- Fernández I, Torrejón E, Rubilar L, Schuffeneger H, Madrid V. Frecuencia de triquinosis humana en Concepción y Arauco, Chile: Estudio en personas autopsiadas en el Servicio Médico Legal de Concepción. Junio de 1996-marzo de 1997. Bol Chil Parasitol 1997; 52: 81-4.

17.- Informe Sanidad Animal Chile. División de Protección Pecuaria Servicio Agrícola y Ganadero, Ministerio de Agricultura, Gobierno de Chile 2013.

18.- Cabrera G, Pinilla N, Dall’Orso L, Parra G. Brote epidémico de triquinosis en Concepción, Chile. Estudio serológico. Bol Chil Parasitol 1982; 37: 47-9.

19.- Martínez L. Martínez C, Martínez L, Grant R. Análisis clínico de 46 pacientes con triquinosis. Parasitol al Día 1986; 10: 52-6. 